\title{
Polyiamonds and Polyhexes with Minimum Site-Perimeter and Achievement Games
}

\author{
Gábor Fülep \\ Lajos Kossuth Bilingual \\ Technical Secondary School \\ Budapest, Hungary \\ fgabci@yahoo.com
}

\author{
Nándor Sieben \\ Department of Mathematics and Statistics \\ Northern Arizona University \\ Flagstaff, Arizona, USA \\ nandor.sieben@nau.edu
}

Submitted: Apr 21, 2008; Accepted: Apr 15, 2010; Published: Apr 30, 2010

Mathematics Subject Classification: 05B50, 91A46

\begin{abstract}
An animal is an edge connected set of finitely many cells of a regular tiling of the plane. The site-perimeter of an animal is the number of empty cells connected to the animal by an edge. The minimum site-perimeter with a given cell size is found for animals on the triangular and hexagonal grid. The formulas are used to show the effectiveness of a simple random strategy in full set animal achievement games.
\end{abstract}

\section{Introduction}

A plane polyform is a figure constructed by joining finitely many congruent basic polygons along their edges. If the basic polygons are cells of a regular tiling of the plane by squares, equilateral triangles or regular hexagons, then the polyform is called a polyomino, polyiamond or polyhex respectively. An animal is a polyomino, polyiamond or polyhex. We only consider animals up to congruence and we allow holes in our animals. The number of cells $s(A)$ of an animal $A$ is called the size of $A$. The standard reference for polyominoes is [5].

Two cells of a regular tiling are adjacent if they share a common edge. The exterior boundary $E(A)$ of the animal $A$ is the set of cells outside of $A$ but adjacent to a cell of $A$. The site-perimeter of $A$ is the number of cells $p(A):=|E(A)|$ in the exterior boundary. In this paper we find formulas for the minimum site-perimeter of polyiamonds and polyhexes with given size. The formula for polyominoes was found in [9].

The motivation partly comes from the importance of the site-perimeter in percolation theory. Similar questions were answered in $[7,8,10,11]$. The site-perimeter is also used in [3] as fixed parameter when counting the number of animals. The motivation also comes from combinatorial game theory. 


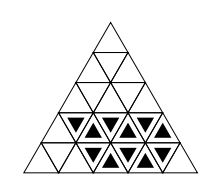

Figure 2.1: The hexagonal animal $T_{3,2,1}^{5}$ cut from an equilateral triangle with edge size 5 .

In a weak animal set $(a, b)$-achievement game two players alternately mark $a$ and $b$ previously unmarked cells using their own colors. The first player (the maker) tries to mark any animal in a given set of target animals. The second player (the breaker) tries to prevent the maker from achieving his goal. Achievement games are studied for example in $[1,2,4,6]$.

If the set of target animals is the set $\mathcal{F}_{s}$ of all animals with size $s$, then the game is called full set achievement game. In this game the maker can follow the strategy of marking random cells adjacent to his earlier marks. We investigate when this strategy can be effective. The answer depends on how small the site-perimeter of animals in $\mathcal{F}_{s}$ can be.

Finding the minimum site-perimeter of an animal with given size is difficult directly. It is easier to find the maximum size of an animal with given site-perimeter because these animals are saturated. A cell $x \in E(A)$ is admissible to $A$ if $p(A \cup x) \leqslant p(A)$. An animal is saturated if it has no admissible cells.

We characterize the saturated polyiamonds in Section 2. This allows us to find the maximum size of polyiamonds with given site-perimeter in Section 3. We find the minimum site-perimeter of polyiamonds with given size in Section 4. The duality between the triangular and hexagonal tilings allows us to quickly translate all these results to polyhexes in Section 5. Finally, we study the random neighbor strategy in Section 6.

\section{Hexagonal polyiamonds}

In this section we characterize the saturated polyiamonds.

Definition 2.1. The polyiamond $T_{a, b, c}^{d}$ gotten from the equilateral triangle polyiamond with edge size $d$ by cutting the corners with edge sizes $a, b$ and $c$ respectively as seen in Figure 2.1 is called a hexagonal polyiamond. We require that $a, b$ and $c$ are nonnegative integers and $d$ is a positive integer. We also require that $a+b, a+c, b+c \leqslant d$.

A hexagonal polyiamond may have sides with zero length. Since congruent animals are considered to be the same, the parametrization is not unique. For example $T_{0,0,0}^{1}=T_{1,1,1}^{2}$. The site-perimeter of a hexagonal polyiamond is equal to its perimeter.

It is easy to see that adding a cell to a hexagonal polyiamond increases the siteperimeter of the animal and so hexagonal polyiamonds are saturated. Our goal is to show that these are the only saturated polyiamonds. 


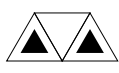

a.

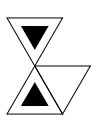

b.

Figure 2.2: The empty cell is admissible to any animal containing the full cells.

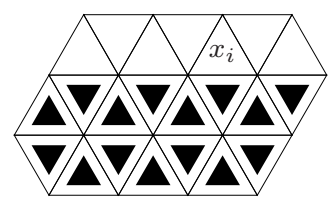

Figure 2.3: An example of the saturation process after adding cell $x_{i}$ to $A_{i-1}=T_{4,1,2}^{6}$. The empty cells must be included in any saturated animal containing $A_{i-1}$ and $x_{i}$. Adding these cells to $A_{i-1}$ results in $A_{i}=T_{3,1,2}^{6}$.

Lemma 2.2. If a polyiamond contains the two full cells but not the empty cell of one of the configurations depicted in Figure 2.2, then the empty cell is admissible.

Proof. In each case, adding the empty cell to the animal decreases the site perimeter by 1 and may increase it by at most 1 .

Roughly speaking, an empty cell at a concave corner of a polyiamond is admissible.

Proposition 2.3. All saturated polyiamonds are hexagonal.

Proof. Let $A$ be a saturated polyiamond. We define an increasing family $A_{1} \subseteq A_{2} \subseteq \cdots \subseteq$ $A_{k}$ of hexagonal subsets of $A$ such that $A_{k}=A$. Let $x_{1}$ be an arbitrary cell of $A$ and define $A_{1}:=\left\{x_{1}\right\}$. If $A_{i-1}=A$ then we are done. Otherwise there is a cell $x_{i} \in A \cap E\left(A_{i-1}\right)$ since $A_{i-1} \subset A$. By symmetry, we can assume that with the parametrization $A_{i-1}=T_{a, b, c}^{d}$ the cell $x_{i}$ is connected to the top edge of $A_{i-1}$ as shown in Figure 2.3. By Lemma 2.2, the set $X_{i}$ of empty cells adjacent to the top edge of $A_{i-1}$ must be a subset of $A$. So the hexagonal polyiamond $A_{i}:=A_{i-1} \cup X_{i}=T_{a-1, b, c}^{d}$ is a subset of $A$. The process ends in finitely many steps since $A$ is finite and $s\left(A_{i-1}\right)<s\left(A_{i}\right)$.

\section{Polyiamonds with fixed site-perimeter and maximum size}

In this section all animals are polyiamonds. Our purpose is to find a formula for $\sigma_{T}(p):=$ $\max \{s(A) \mid p(A)=p\}$.

Lemma 3.1. For each polyiamond $A$ there is a polyiamond $\tilde{A}$ such that $A \subseteq \tilde{A}$ and $p(\tilde{A})=p(A)+1$. 


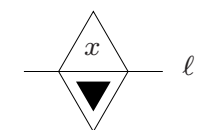

a. $\ell$ contains a cell edge

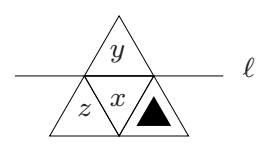

b. $\ell$ contains no cell edges

Figure 3.1: The site-perimeter of a polyiamond $A$ can be incremented by adding either cell $x$ or the cells in $\{x, y\}$ to $A$. The site-perimeter of $A$ does not increase if the full cell is removed from $A$.

Proof. Let $\ell$ be the horizontal line that touches $A$ but has no cells of $A$ above it. Such $\ell$ exists since $A$ is finite. If $\ell$ contains the edge of a cell of $A$ as shown in Figure 3.1.a, then adding cell $x$ to $A$ increases the site-perimeter of $A$ by 1 .

If $\ell$ contains none of the cell edges of $A$ as shown in Figure 3.1.b, then cell $x$ does not belong to $A$. If $z \notin E(A)$ then $p(A \cup\{x\})=p(A)+1$. If $z \in E(A)$ then $p(A \cup\{x, y\})=$ $p(A)+1$.

Proposition 3.2. If $p(A)=p$ and $s(A)=\sigma_{T}(p)$, then $A$ is saturated.

Proof. Suppose that $A$ is not saturated. Let $\tilde{A}:=A \cup\{x\}$ where $x$ is a cell admissible to $A$. Then $p(\tilde{A}) \leqslant p$. If $p(\tilde{A})<p$ then by Lemma 3.1, we can add cells to $\tilde{A}$ until its site-perimeter reaches $p$. This is a contradiction since then $A$ cannot have maximum size.

The following convenient formula is the consequence of the choice of the parameters in Definition 2.1.

Lemma 3.3. For each hexagonal polyiamond $T_{a, b, c}^{d}$ we have

$$
p\left(T_{a, b, c}^{d}\right)^{2}-6 s\left(T_{a, b, c}^{d}\right)=3(d-a-b-c)^{2}+2\left((a-b)^{2}+(a-c)^{2}+(b-c)^{2}\right) .
$$

Proof. It is easy to see that $p\left(T_{a, b, c}^{d}\right)=3 d-a-b-c$ and $s\left(T_{a, b, c}^{d}\right)=d^{2}-a^{2}-b^{2}-c^{2}$. The result follows from these facts after a short calculation.

Proposition 3.4. Let $p=6 k+r$ where $0 \leqslant r<6$. Then

$$
\sigma_{T}(p)=\left\{\begin{array}{ll}
s\left(T_{k, k, k}^{\left\lfloor\frac{p}{2}\right\rfloor}\right), & r \in\{0,3\} \\
s\left(T_{k}^{\left\lfloor\frac{p}{2}\right\rfloor}\right), k, k & r=1 \\
s\left(T_{k, k}^{\left\lfloor\frac{p}{2}\right\rfloor}\right), & r \in\{2,5\} \\
s\left(T_{k, k+1, k+1}^{\left\lfloor\frac{p}{2}\right\rfloor}\right), & r=4
\end{array} .\right.
$$

Proof. We know that $\sigma_{T}(p)=s\left(T_{a, b, c}^{d}\right)$ for some saturated animal $T_{a, b, c}^{d}$ with $p\left(T_{a, b, c}^{d}\right)=p$. Then $d=(p+a+b+c) / 3$. We see from Lemma 3.3 that the maximum of $s\left(T_{a, b, c}^{d}\right)$ is reached when

$$
M:=p^{2}-6 s\left(T_{a, b, c}^{d}\right)=3(d-a-b-c)^{2}+2\left((a-b)^{2}+(a-c)^{2}+(b-c)^{2}\right)
$$


is as small as possible. Note that $M \equiv_{6} p^{2}$ and $M$ is nonnegative since it is the sum of squares. We need to consider several cases.

If $r=0$ then let $a=b=c=k$. Then $d=3 k$ and so $M=0$. This is clearly the minimum.

If $r=1$ then let $a=k-1$ and $b=c=k$. Then $d=3 k$ and so $M=7$. Since $M \equiv_{6} 1$, the only possibly smaller value for $M$ is 1 . The equation $1=3 p+2 q$ has no nonnegative integer solution so the minimum of $M$ is 7 .

If $r=2$ then let $a=b=k$ and $c=k+1$. Then $d=3 k+1$ and so $M=4$. Since $M \equiv{ }_{6} 4$, the minimum of $M$ is 4 .

If $r=3$ then let $a=b=c=k$. Then $d=3 k+1$ and so $M=3$. Since $M \equiv_{6} 3$, the minimum of $M$ is 3 .

If $r=4$ then let $a=k$ and $b=c=k+1$. Then $d=3 k+2$ and so $M=4$. Since $M \equiv_{6} 4$, the minimum of $M$ is 4 .

If $r=5$ then let $a=b=k$ and $c=k+1$. Then $d=3 k+2$ and so $M=7$. Since $M \equiv_{6} 1$, the only possibly smaller value for $M$ is 1 . The equation $1=3 p+2 q$ has no nonnegative integer solution so the minimum of $M$ is 7 .

Figure 3.2 shows the polyiamond families chosen in the previous theorem.

Proposition 3.5. For $p \geqslant 3$ we have $\sigma_{T}(p)=2\left\lfloor p^{2} / 12-p / 2\right\rfloor+p$.

Proof. Let $p=6 k+r \geqslant 3$ where $0 \leqslant r<6$. Then Proposition 3.4 and the formula $s\left(T_{a, b, c}^{d}\right)=d^{2}-a^{2}-b^{2}-c^{2}$ imply that $\sigma_{T}(p)=6 k^{2}+2 r k+|r-1|-1$. Substituting $p=6 k+r$ into this formula gives

$$
\sigma_{T}(p)-\left(2\left\lfloor p^{2} / 12-p / 2\right\rfloor+p\right)=|r-1|-r-1-2\left\lfloor r^{2} / 12-r / 2\right\rfloor .
$$

It is easy to check that this expression is 0 for all $r \in\{0, \ldots, 5\}$.

Proposition 3.6. The function $\sigma_{T}$ is strictly increasing on its domain $\{3,4, \ldots\}$.

Proof. First, suppose that $p=6 k+r$ where $0 \leqslant r<5$. Then $\sigma_{T}(p+1)-\sigma_{T}(p)=$ $2 k+|r|-|r-1|$. If $k=0$ then $r \geqslant 3$ and so $2 k+|r|-|r-1|=1$. If $k \geqslant 1$ then $2 k+(|r|-|r-1|) \geqslant 2 k-1 \geqslant 1$.

Now suppose that $p=6 k+5$. Then $\sigma_{T}(p+1)-\sigma_{T}(p)=6(k+1)^{2}-\left(6 k^{2}+10 k+3\right)=$ $2 k+3 \geqslant 1$.

\section{Polyiamonds with fixed size and minimum site- perimeter}

In this section all animals are polyiamonds. Our purpose is to find a formula for $\pi_{T}(s):=$ $\min \{p(A) \mid s(A)=s\}$.

Lemma 4.1. For all $s \geqslant 1$ we have $\sigma_{T}\left(\pi_{T}(s)\right) \geqslant s$. 


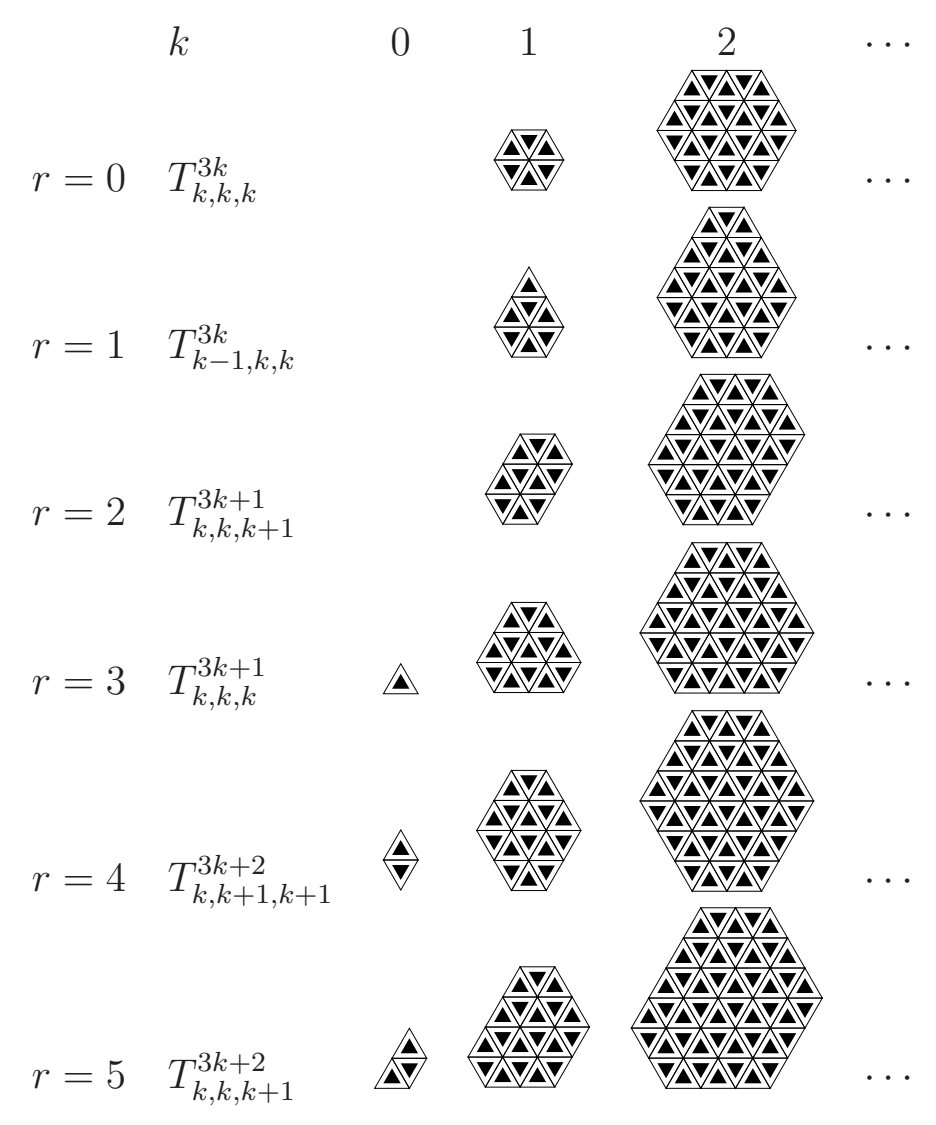

Figure 3.2: Maximum size animals with fixed site-perimeter $p=6 k+r$. Note that adding an extra layer of cells around an animal creates the next animal in the row. 
Proof. Let $p:=\pi_{T}(s)$. Then there is a polyiamond $A$ with $s(A)=s$ and $p(A)=p$. Hence $\sigma_{T}(p) \geqslant s$.

Lemma 4.2. For all $p \geqslant 3$ we have $\pi_{T}\left(\sigma_{T}(p)\right)=p$.

Proof. Let $s:=\sigma_{T}(p)$. Then there is a polyiamond $A$ with $p(A)=p$ and $s(A)=s$. Hence $\pi_{T}(s) \leqslant p$. If $q<p$ then $\sigma_{T}(q)<\sigma_{T}(p)=s$ by Proposition 3.6, and so no polyiamond with site-perimeter $q$ can have size $s$. Thus $\pi_{T}(s) \geqslant p$.

Lemma 4.3. For all polyiamond $A$ with $s(A) \geqslant 2$ there is a subset $\hat{A} \subset A$ such that $s(\hat{A})=s(A)-1$ and $p(\hat{A}) \leqslant p(A)$.

Proof. We show that if $s(A) \geqslant 2$ then we can remove a cell from $A$ without increasing its site-perimeter. Let $\ell$ be the horizontal line that touches $A$ but has no cells of $A$ above it, as in the proof of Lemma 3.1. Let $w$ be the leftmost cell of $A$ that touches $\ell$. The full cell in Figure 3.1 represents the two possible positions of $w$ with respect to $\ell$. It is easy to see that, in both cases, cell $x$ is adjacent to $w$ but it is not adjacent to any other cell of $A$. So removing $w$ from $A$ decreases the site-perimeter by at least 1 since $x$ falls out of the exterior boundary. Since $A$ is connected, $A$ must have a cell adjacent to $w$. So the removal also increases the site-perimeter by exactly 1 since $w$ becomes a member of the site perimeter. So $\hat{A}=A \backslash\{w\}$ satisfies the requirements.

Proposition 4.4. The function $\pi_{T}$ is increasing.

Proof. For all $s \geqslant 2$ there is a polyiamond $A$ with $s(A)=s$ and $p(A)=\pi_{T}(s)$. Let $\hat{A}$ be the subset of $A$ guaranteed by the previous Lemma. Then $s(\hat{A})=s-1$ and so $\pi_{T}(s-1) \leqslant p(\hat{A}) \leqslant p(A)=\pi_{T}(s)$.

Proposition 4.5. For all $s \geqslant 1$ we have $\pi_{T}(s)=\min \left\{p \mid \sigma_{T}(p) \geqslant s\right\}$.

Proof. Since $s \leqslant \sigma_{T}\left(\pi_{T}(s)\right), q:=\min \left\{p \mid \sigma_{T}(p) \geqslant s\right\}$ exists. If $q=3$ then clearly $s=1$ and the statement is true, so we can assume that $q>3$. Then $\sigma_{T}(q-1)<s \leqslant \sigma_{T}(q)$ and so by Lemma 4.2 and Proposition 4.4 we have

$$
q-1=\pi_{T}\left(\sigma_{T}(q-1)\right) \leqslant \pi_{T}(s) \leqslant \pi_{T}\left(\sigma_{T}(q)\right)=q .
$$

Equality on the first inequality is impossible since $q-1=\pi_{T}(s)$ and Lemma 4.1 imply the contradiction $s>\sigma_{T}(q-1)=\sigma_{T}\left(\pi_{T}(s)\right) \geqslant s$. So we must have $\pi_{T}(s)=q$.

If $p$ is a statement, then we define $[p]$ to be 1 if $p$ is true and 0 if $p$ is false. For example, $[i=j]$ is the Kronecker delta $\delta_{i, j}$. The next result is one of our main theorems.

Theorem 4.6. For all $s \geqslant 1$ we have

$$
\pi_{T}(s)=\lceil\sqrt{6 s}\rceil+\left\lceil\lceil\sqrt{6 s}\rceil \equiv_{6} \pm 1\right] \cdot[\lceil\sqrt{6 s}\rceil \neq\lceil\sqrt{6 s+6}\rceil] .
$$




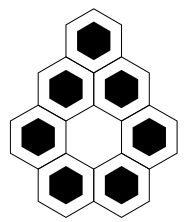

$B$

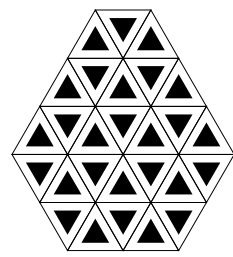

$B^{\sharp}$

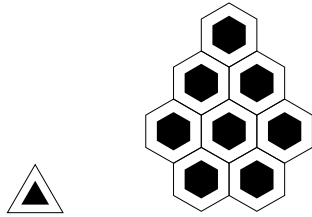

$\left(B^{\sharp}\right)^{b}$

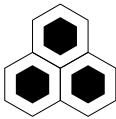

$\left(B^{b}\right)^{\#}$

Figure 5.1: A polyhex and its various duals.

Proof. We know that $\sigma_{T}(p)=2\left\lfloor p^{2} / 12-p / 2\right\rfloor+p$. It is easy to see that $\sigma_{T}(p)=\left\lfloor p^{2} / 6-\right.$ $p-1\rfloor+p$ if the remainder of $p$ is 1 or 5 modulo 6 , and $\sigma_{T}(p)=\left\lfloor p^{2} / 6-p\right\rfloor+p$ otherwise. Let $X:=\left\{n \in \mathbf{N} \mid n \equiv_{6} \pm 1\right\}$ and $Y:=\mathbf{N} \backslash X$. Then

$$
\begin{aligned}
\pi_{T}(s) & =\min \left\{p \mid \sigma_{T}(p) \geqslant s\right\} \\
& =\min \left(\left\{p \in X \mid\left\lfloor p^{2} / 6-p-1\right\rfloor+p \geqslant s\right\} \cup\left\{p \in Y \mid\left\lfloor p^{2} / 6-p\right\rfloor+p \geqslant s\right\}\right) \\
& =\min \left(\left\{p \in X \mid p^{2} / 6-1 \geqslant s\right\} \cup\left\{p \in Y \mid p^{2} / 6 \geqslant s\right\}\right) \\
& =\min (\{p \in X \mid p \geqslant \sqrt{6 s+6}\} \cup\{p \in Y \mid p \geqslant \sqrt{6 s}\}) \\
& =\min (\{p \in X \mid p \geqslant\lceil\sqrt{6 s+6} \mid\} \cup\{p \in Y \mid p \geqslant\lceil\sqrt{6 s}\rceil\}) .
\end{aligned}
$$

So if $\lceil\sqrt{6 s}\rceil \in Y$ or $\lceil\sqrt{6 s+6}\rceil=\lceil\sqrt{6 s}\rceil$ then $\pi_{T}(s)=\lceil\sqrt{6 s}\rceil$. Otherwise $\pi_{T}(s)=$ $\lceil\sqrt{6 s+6}\rceil=\lceil\sqrt{6 s}\rceil+1$ since it is easy to see that $\lceil\sqrt{6 s+6}\rceil-\lceil\sqrt{6 s}\rceil \leqslant 1$ for all $s \geqslant 1$.

\section{Polyhexes}

The dual of a regular tiling is constructed by drawing line segments connecting the center points of all pairs of adjacent cells. The dual of a tiling by regular hexagons is a tiling by equilateral triangles.

Definition 5.1. Let $B$ be an animal. The outer dual of $B$ is the animal $B^{\sharp}$ built from those cells of the dual tiling that intersect $B$. The inner dual of $B$ is the animal $B^{b}$ built from those cells of the dual tiling that are inside $B$.

It is easy to see that the duals of an animal are connected through edges so they are in fact animals. The duals of a polyhex are polyiamond and the duals of a polyiamond are polyhexes. Figure 5.1 shows a polyhex and its duals. Note that $\left(B^{\sharp}\right)^{b}$ and $\left(B^{b}\right)^{\sharp}$ are not $B$ in general.

We can find the answer to our question about polyhexes from our results about polyiamonds using this dual connection. The following is an easy consequence of the definitions.

Definition 5.2. A polyhex $B$ is called hexagonal if $B^{\sharp}$ is a hexagonal polyiamond. If $B^{\sharp}=T_{a, b, c}^{d}$ then $B$ is denoted by $H_{a, b, c}^{d}$. 


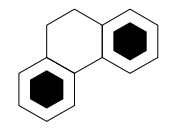

Figure 5.2: The empty cell is admissible to any animal containing the full cells.

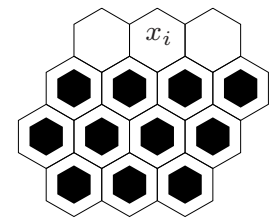

$B_{i}=H_{3,2,3}^{8}$

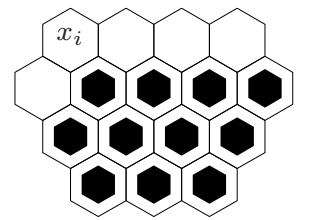

$B_{i}=H_{4,3,3}^{9}$

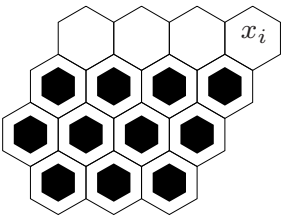

$B_{i}=H_{4,2,4}^{9}$

Figure 5.3: The three possible saturation processes after adding cell $x_{i}$ to $B_{i-1}=H_{4,2,3}^{8}$. The empty cells must be included in any saturated animal containing $B_{i-1}$ and $x_{i}$. Adding these cells to $B_{i-1}$ results in $B_{i}$.

The reader can easily verify the following result.

Lemma 5.3. If $B$ is a hexagonal polyhex, then $p(B)=p\left(B^{\sharp}\right)$.

It is easy to see that adding a cell to a hexagonal animal increases the site-perimeter of the animal and so hexagonal animals are saturated.

Lemma 5.4. If a polyhex contains the two full cells but not the empty cell of the configuration depicted in Figure 5.2, then the empty cell is admissible.

Proof. Adding the empty cell to the animal decreases the site perimeter by 1 and may increase it by at most 1 .

Proposition 5.5. All saturated polyhexes are hexagonal.

Proof. The argument uses Lemma 5.4 and is similar to the proof of Proposition 2.3. Figure 5.3 shows the saturation process after adding cell $x_{i}$ to the hexagonal polyhex $B_{i-1}$.

Lemma 5.6. For each polyhex $B$ with size at least 2 there is a polyhex $\tilde{B}$ such that $B \subseteq \tilde{B}$ and $p(\tilde{B})=p(B)+1$.

Proof. Let $U$ be the horizontal row of cells that contains the highest cells of $B$. We need to consider several cases shown in Figure 5.4. If there are two adjacent cells $x, y \in B \cup U$, then $\tilde{B}=B \cup\{u\}$ as shown in case 1 .

If there are no such cells, then let $x$ be an arbitrary cell in $U$. Since $B$ has at least 2 cells, $B$ must have a cell $w$ adjacent to $x$. Let $y \in U$ be the cell adjacent to both $x$ and $w$. So we are in the situation shown on the left picture of Figure 5.4. Now we have three 


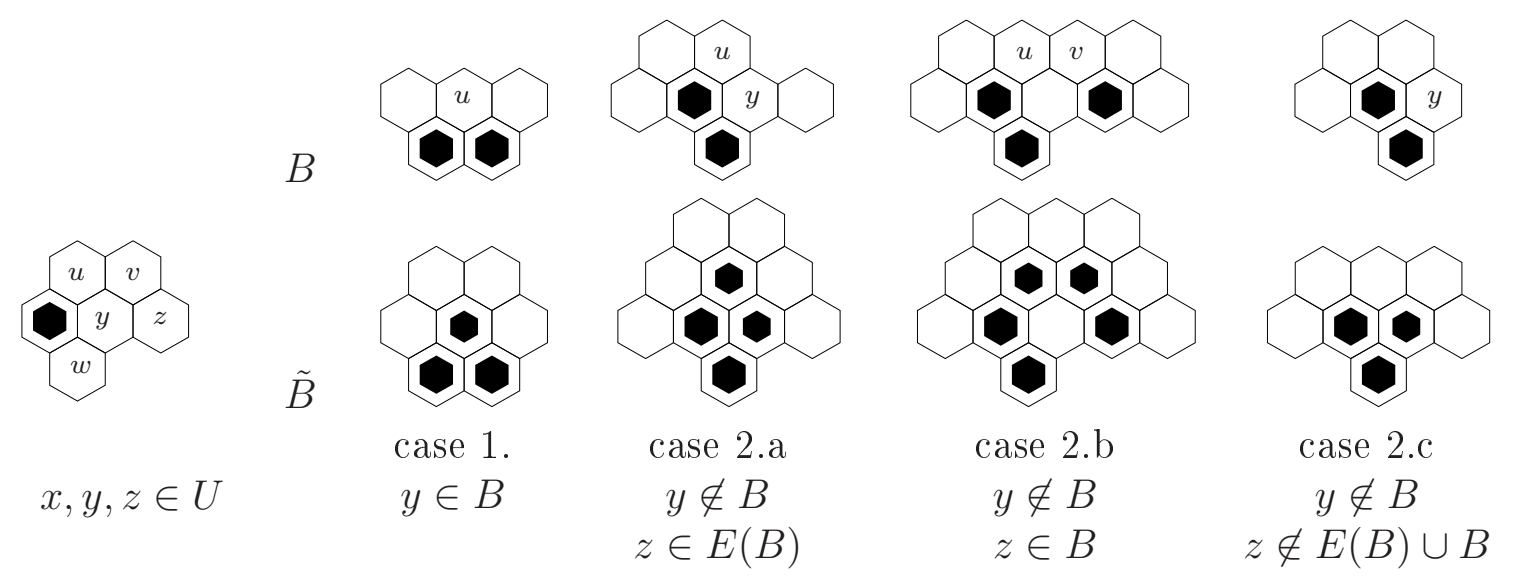

Figure 5.4: Incrementing the site-perimeter of the polyhex $B$. The picture on the left shows the cell $x$ and the notation for its neighbors. The pictures on the right show $B$ and $\tilde{B}$. The known exterior boundary cells are shown as empty cells. The cells on the top row with letters in them are in $\tilde{B} \backslash B$. They become the smaller full cells on the bottom row.

cases depending on cell $z$. If $z \in E(B)$ then $\tilde{B}=B \cup\{u, y\}$ as shown in case 2.a. If $z \in B$ then $\tilde{B}=B \cup\{u, v\}$ as shown in case 2.b. Finally, if $z \notin E(B) \cup B$ then $\tilde{B}=B \cup\{y\}$ as shown in case 2.c.

Proposition 5.7. If $p(B)=p$ and $s(B)=\sigma_{T}(p)$, then $B$ is saturated.

Proof. The proof uses Lemma 5.6. It is essentially the same as that of Proposition 3.2.

Proposition 5.8. If $B$ is a hexagonal polyhex, then $s\left(B^{\sharp}\right)=2 s(B)+p(B)-2$.

Proof. We use Euler's formula for the planar graph built from the vertices and edges of the cells of $B^{\sharp}$. The number of vertices is $v\left(B^{\sharp}\right)=s(B)+p(B)$. The number of faces is $f\left(B^{\sharp}\right)=s\left(B^{\sharp}\right)+1$. The number of edges $e\left(B^{\sharp}\right)$ satisfy the equation $e\left(B^{\sharp}\right)=$ $3 s\left(B^{\sharp}\right)-\left(e\left(B^{\sharp}\right)-p\left(B^{\sharp}\right)\right)$ which gives $2 e\left(B^{\sharp}\right)=3 s\left(B^{\sharp}\right)+p\left(B^{\sharp}\right)$. From here Euler's formula $v\left(B^{\sharp}\right)+f\left(B^{\sharp}\right)=e\left(B^{\sharp}\right)+2$ gives

$$
2 s(B)+2 p(B)+2 s\left(B^{\sharp}\right)+2=3 s\left(B^{\sharp}\right)+p\left(B^{\sharp}\right)+4
$$

which simplifies to the desired equality.

Proposition 5.9. For $p \in \mathbf{N} \backslash\{1,2,3,4,5,7\}$ we have $\sigma_{T}(p)=2 \sigma_{H}(p)+p-2$.

Proof. Let $B$ be a hexagonal polyhex such that $p(B)=p$ and $s(B)=\sigma_{H}(p)$. Since $B$ is hexagonal, $B^{\sharp}$ is also hexagonal and so $p\left(B^{\sharp}\right)=p$. Then

$$
\sigma_{T}(p) \geqslant s\left(B^{\sharp}\right)=2 s(B)+p(B)-2=2 \sigma_{H}(p)+p-2 .
$$




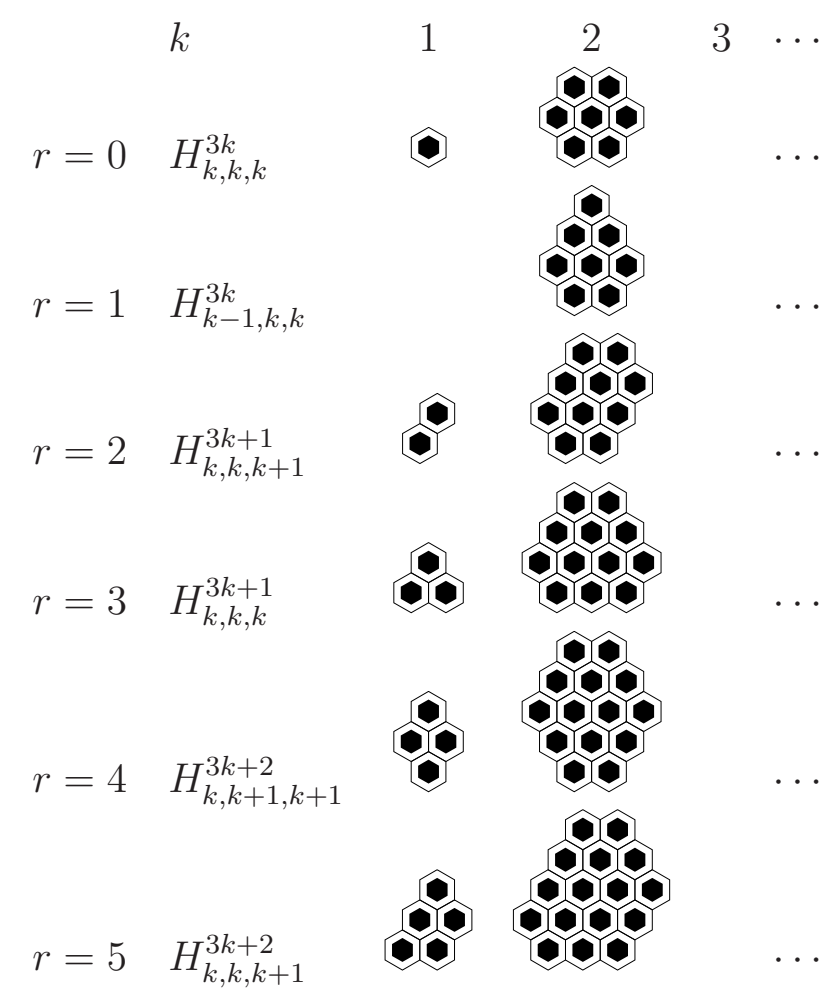

Figure 5.5: Maximum size polyhexes with fixed site-perimeter $p=6 k+r$. Note that adding an extra layer of cells around an animal creates the next animal in the row. 
For the other direction let $A$ be the hexagonal polyiamond chosen in Proposition 3.4 such that $p(A)=p$ and $s(A)=\sigma_{T}(p)$. It is easy to see that because of the choice of $p$ the dual $B:=A^{\text {b }}$ satisfies $B^{\sharp}=A$. Hence $p(B)=p$ and so

$$
\sigma_{H}(p) \geqslant s(B)=(s(A)-p(B)+2) / 2=\left(\sigma_{T}(p)-p+2\right) / 2 .
$$

Figure 5.5 shows the polyhex families that realize the $\sigma_{H}$ values.

Proposition 5.10. For $p \in \mathbf{N} \backslash\{1,2,3,4,5,7\}$ we have $\sigma_{H}(p)=\left\lfloor p^{2} / 12-p / 2\right\rfloor+1$.

Proof. The proof follows from the calculation

$$
\sigma_{H}(p)=\left(\sigma_{T}(p)-p+2\right) / 2=\left(2\left\lfloor p^{2} / 12-p / 2\right\rfloor+p-p+2\right) / 2=\left\lfloor p^{2} / 12-p / 2\right\rfloor+1 .
$$

The proof of the following proposition is essentially the same as that of Proposition 4.5.

Proposition 5.11. For all $s \geqslant 1$ we have $\pi_{H}(s)=\min \left\{p \mid \sigma_{H}(p) \geqslant s\right\}$.

The following is one of our main theorems. The proof is an easier version of the the proof of Theorem 4.6.

Theorem 5.12. For all $s \geqslant 1$ we have $\pi_{H}(s)=\lceil\sqrt{12 s-3}\rceil+3$.

Proof. The result follows form the calculation

$$
\begin{aligned}
\pi_{H}(s) & =\min \left\{p \mid \sigma_{H}(p) \geqslant s\right\}=\min \left\{p \mid\left\lfloor p^{2} / 12-p / 2\right\rfloor+1 \geqslant s\right\} \\
& =\min \left\{p \mid p^{2} / 12-p / 2+1 \geqslant s\right\}=\min \left\{p \mid(p-3)^{2} \geqslant 12 s-3\right\} \\
& =\min \{p \mid p \geqslant \sqrt{12 s-3}+3\}=\lceil\sqrt{12 s-3}\rceil+3 .
\end{aligned}
$$

\section{The random neighbor strategy}

Now we return to achievement games described in the Introduction. In a full set $(a, b)$ achievement game, the maker can follow the very simple strategy of randomly marking cells adjacent to any of his earlier marks. We call this the random neighbor strategy. If the maker is able to follow this strategy for $s$ turns, then he can mark an animal of size $s a$ and win the $\mathcal{F}_{s a}$-achievement game.

Of course it is possible that this strategy fails after $r<s$ turns because the whole exterior boundary $E\left(P_{r a}\right)$ of the animal $P_{r a} \in \mathcal{F}_{r a}$ built from the maker's $r a$ marks is already marked by the breaker. This will not happen though if the total number $r b$ of marks by the breaker is smaller than the smallest possible site-perimeter of $P_{r a}$.

In this section we use $\pi$ to denote either $\pi_{T}$ or $\pi_{H}$. 


\begin{tabular}{|c|c|c|c|c|}
\hline$S_{T}(a, b)$ & $b=1$ & $b=2$ & $b=3$ & $b=4$ \\
\hline$a=1$ & 6 & 2 & 1 & 1 \\
\hline$a=2$ & 12 & 3 & 2 & 1 \\
\hline$a=3$ & 18 & 5 & 2 & 2 \\
\hline$a=4$ & 24 & 6 & 3 & 2 \\
\hline
\end{tabular}

a. polyiamonds

\begin{tabular}{|c|c|c|c|c|}
\hline$S_{H}(a, b)$ & $b=1$ & $b=2$ & $b=3$ & $b=4$ \\
\hline$a=1$ & 18 & 6 & 3 & 2 \\
\hline$a=2$ & 30 & 9 & 5 & 3 \\
\hline$a=3$ & 42 & 12 & 6 & 4 \\
\hline$a=4$ & 54 & 15 & 8 & 5 \\
\hline
\end{tabular}

b. polyhexes

Table 1: The maximum number of turns for which the random neighbor strategy is successful in the $(a, b)$-achievement game. The values are calculated using the formula of Proposition 6.2 and Table 2.

Proposition 6.1. The random neighbor strategy is successful in the $(a, b)$-achievement game for $s$ turns if $r b<\pi(r a)$ for all $r<s$.

Proof. Let $A_{t} \in \mathcal{F}_{t}$ be the animal marked by the maker after $t$ of his marks. The strategy clearly works in the first turn. Suppose that the strategy works for $r<s$ terms. After the $r$-th turn the breaker marked $r b$ cells. During the next turn, the exterior perimeter of $A_{t}$ satisfies the inequality $p\left(A_{t}\right) \geqslant \pi(t) \geqslant \pi(r a)$. Since the exterior perimeter is larger than the total number of cells marked by the breaker, the maker can always find a cell in the exterior boundary of $A_{t}$ for his next mark.

Note that this result also holds for polyominoes. Let $S_{H}(a, b):=\max \{s \mid(\forall r<s) r b<$ $\left.\pi_{H}(r a)\right\}$.

Proposition 6.2. Let $a$ and $b$ be positive integers. If $a<b(1 / \sqrt{3}-1 / 2)$ then $S_{H}(a, b)=1$ otherwise $S_{H}(a, b)=\left\lceil\left(6 a+3 b+\sqrt{36 a^{2}+36 a b-3 b^{2}}\right) / b^{2}\right\rceil$.

Proof. For $a, b, r \in \mathbf{N}$ we have

$$
\begin{aligned}
r b<\pi_{H}(r a) & \Leftrightarrow r b<\lceil\sqrt{12 r a-3}\rceil+3 \\
& \Leftrightarrow r b<\sqrt{12 r a-3}+3 \\
& \Leftrightarrow r b<3 \text { or } b^{2} r^{2}-(6 b+12 a) r+12<0 .
\end{aligned}
$$

The roots of $b^{2} r^{2}-(6 b+12 a) r+12=0$ for $r$ are $\left(6 a+3 b \pm \sqrt{36 a^{2}+36 a b-3 b^{2}}\right) / b^{2}$. It is easy to see that for $a \geqslant b(1 / \sqrt{3}-1 / 2)$ these roots are real, the smaller root is less than 1 , and the larger root is greater than $3 / b$.

It is possible to develop a formula for the similarly defined $S_{T}(a, b)$ in the polyiamond case. Since the formula for $\pi_{T}$ is fairly complicated, the result is not worth the effort. Table 1 lists some values for $S_{T}$ and $S_{H}$. 


\begin{tabular}{|c|c|c|c|c|c|c|c|c|c|c|c|c|c|c|c|c|c|c|}
\hline$s$ & 1 & 2 & 3 & 4 & 5 & 6 & 7 & 8 & 9 & 10 & 11 & 12 & 13 & 14 & 15 & 16 & 17 & 18 \\
\hline$\pi_{T}(s)$ & 3 & 4 & 5 & 6 & 6 & 6 & 7 & 8 & 8 & 8 & 9 & 9 & 9 & 10 & 10 & 10 & 11 & 11 \\
\hline$\pi_{H}(s)$ & 6 & 8 & 9 & 10 & 11 & 12 & 12 & 13 & 14 & 14 & 15 & 15 & 16 & 16 & 17 & 17 & 18 & 18 \\
\hline
\end{tabular}

Table 2: Some values for $\pi_{T}$ and $\pi_{H}$. The italic numbers correspond to the hexagonal animals in Figure 3.2 and Figure 5.5.

\section{References}

[1] Jens-P. Bode and Heiko Harborth. Hexagonal polyomino achievement. Discrete Math., 212(1-2):5-18, 2000. Graph theory (Dörnfeld, 1997).

[2] Jens-P. Bode and Heiko Harborth. Triangle polyomino set achievement. In Proceedings of the Thirty-second Southeastern International Conference on Combinatorics, Graph Theory and Computing (Baton Rouge, LA, 2001), volume 148, pages 97-101, 2001.

[3] M.P. Delest, D. Gouyou-Beauchamps, and B. Vauquelin. Enumeriation of parallelogram polyominoes with given bond and site perimeter. Graphs Comb., 3:325-339, 1987.

[4] Martin Gardner. Mathematical games. Sci. Amer., 240:18-26, 1979.

[5] Solomon G. Golomb. Polyominoes: Puzzles, Patterns, Problem and Packings. Princeton University Press, 1965.

[6] Frank Harary. Achievement and avoidance games for graphs. In Graph theory (Cambridge, 1981), volume 13 of Ann. Discrete Math., pages 111-119. North-Holland, Amsterdam, 1982.

[7] Frank Harary and Heiko Harborth. Extremal animals. J. Combinatorics Information Syst. Sci., 1(1):1-8, 1976.

[8] T. Prellberg and A.L. Owczarek. On the asymptotics of the finite-perimeter partition function of two-dimensional lattice vesicles. Commun. Math. Phys., 201(3):493-505, 1999.

[9] Nándor Sieben. Polyominoes with minimum site-perimeter and full set achievement games. European Journal of Combinatorics, 29:108-117, 2008.

[10] L.M. Stratychuk and C.E. Soteros. Statistics of collapsed lattice animals: Rigorous results and Monte Carlo simulations. J. Phys. A, Math. Gen., 29(22):7067-7087, 1996.

[11] Winston C. Yang. Adding layers to bumped-body polyforms with minimum perimeter preserves minimum perimeter. Electron. J. Comb., 13(1):R6, 11p, 2006. 